\title{
La situation linguistique dans la république du Mari-El
}

\section{Zoja Zorina}

Traducteur : Vincent Lorenzini

\section{OpenEdition Journals}

\section{Édition électronique}

URL : https://journals.openedition.org/efo/2322

DOI : $10.4000 /$ efo. 2322

ISSN : 2275-1947

Éditeur

INALCO

\section{Édition imprimée}

Date de publication : 1 décembre 2013

ISBN : 978-2-343-04446-0

ISSN : 0071-2051

\section{Référence électronique}

Zoja Zorina, « La situation linguistique dans la république du Mari-El », Études finno-ougriennes [En ligne], 45 | 2013, mis en ligne le 05 février 2015, consulté le 08 juillet 2021. URL : http:// journals.openedition.org/efo/2322 ; DOI : https://doi.org/10.4000/efo.2322

Ce document a été généré automatiquement le 8 juillet 2021.

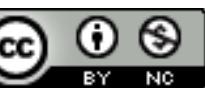

Études finno-ougriennes est mis à disposition selon les termes de la Licence Creative Commons Attribution - Pas d'Utilisation Commerciale 4.0 International. 


\title{
La situation linguistique dans la république du Mari-El
}

\author{
Zoja Zorina \\ Traduction : Vincent Lorenzini
}

1 La situation sociolinguistique en Russie est très complexe, en raison des nombreux peuples qui l'habitent et parlent des langues différentes; il faut en particulier souligner les problèmes auxquels est confronté un peuple numériquement petit comme les Maris des collines.

2 Selon le recensement de la population établi dans toute la Russie en 2010, la république du Mari El compte aujourd'hui 692400 habitants. La répartition par nationalités est la suivante : 313900 Russes, 290900 Maris (dont 23500 Maris des collines), 38400 Tatars, etc. ${ }^{1}$ Par « Maris », il faut entendre les Maris des collines et les Maris des plaines qui vivent sur le territoire de la république du Mari El.

3 En raison de son caractère très ancien et de l'absence de sources authentiques léguées par l'histoire, l'apparition des Maris des collines et des Maris des plaines est difficile à dater. Nous savons toutefois qu'aux $\mathrm{Xv}^{\mathrm{e}}$-XvI $\mathrm{X}^{\mathrm{e}}$ siècles la division en deux ethnies existait déjà, comme en témoignent des sources littéraires de l'époque : «Il se trouve dans l'oblast de Kazan' deux groupes de Tchérémisses [...], l'un est sis de ce côté de la Volga, au milieu de hautes collines, le long des vallées, et on l'appelle "des collines»; le groupe habitant les plaines se trouve de l'autre côté de la Volga et est appelé «des plaines » (Osnovy 1976, p. 45). Dès le début du xix ${ }^{e}$ siècle, bien avant la codification des langues maries, les chercheurs étrangers avaient noté dans leurs travaux les différences considérables qui existaient entre les deux langues (Asylbaev 1951, Castrén 1945, Lewis 2009, Ramstedt 1902).

4 Le premier monument littéraire en mari des collines date de 1769 (Duhovnaja, p. 45). En 1821 fut publié à Saint-Pétersbourg "L'Évangile dans le dialecte des collines" (Evangelie 1821). En 1812, dans le village de Pertnury (raïon des Maris des collines), le prêtre Andrej Danilovič Al'binskij ouvrit dans sa propre maison la première école marie (Wiedemann 1847). En 1813, A. D. Al'binskij rédigea une "Grammaire de la langue tchérémisse des collines », qui fut publiée en 1837 à Kazan' sous le nom de «Grammaire 
tchérémisse ». Tirée à 1200 exemplaires, chiffre considérable pour l'époque (Al'binskij 1837), la grammaire servait de manuel d'apprentissage pour les Maris des écoles des districts de Kazan' et de Čeboksary.

5 En 1867 fut publié le premier abécédaire mari, intitulé "Méthode simplifiée d'apprentissage pour les enfants tchérémisses des villages des collines" (Wiedemann 1947). Son auteur était le diacre Ioann Kedrov, instituteur à l'école paroissiale de Čermyševskij du district de Koz'modem'jansk (raïon des Maris des collines). En 1870 parut le premier abécédaire dans le dialecte des plaines, intitulé "Méthode simplifiée d'apprentissage pour les enfants tchérémisses des villages des plaines ", écrit par Unjžinskij, professeur de l'école autochtone (Wiedemann 1847). Par la suite, de 1867 jusqu'au début du Xx $x^{e}$ siècle, vingt autres abécédaires virent le jour. Au total, jusqu'à la révolution de 1917, plus de 250 livres furent publiés dans les langues maries : abécédaires, dictionnaires, calendriers, littérature ecclésiastique.

6 Après la révolution de 1917, des efforts furent entrepris pour créer une seule langue littéraire pour tous les Maris, mais les différences trop grandes entre les deux langues maries ne permirent pas d'atteindre ce but. À ce propos on ne peut que réfuter l'opinion de certains auteurs, par exemple A.J. Musorin, qui déclarait: "Il n'y a manifestement aucune raison de considérer les deux variantes littéraires du mari celle des collines et celle des plaines - comme des langues distinctes : l'une comme l'autre sont nées de la codification de différents dialectes de la langue marie» (Musorin 2011). En premier lieu, le mari des collines et le mari des plaines ne peuvent être appelés "variantes ", car cela supposerait la primauté de l'une sur l'autre. Or l'histoire ne nous permet pas de dire laquelle des deux langues occupe la première place. De par son système vocalique et consonantique plus riche, le mari des collines pourrait d'ailleurs bien prétendre à cette primauté. En second lieu, la naissance des langues maries n'est pas le résultat d'une codification: c'est au contraire parce qu'il existait deux langues différentes que la standardisation a été possible et nécessaire. Il ne fait aucun doute que le mari des collines et le mari des plaines sont des langues très apparentées. Toutefois, au cours de l'histoire, elles ont évolué relativement l'une à l'autre (se sont-elles éloignées ou rapprochées ?) et sont devenues indépendantes. Si le vieux russe a donné naissance à des langues comme le biélorusse, le russe et l'ukrainien, pourquoi le tchérémisse n'aurait-il pas pu engendrer le mari des collines et le mari des plaines?

7 On sait bien que le statut d'une langue dépend de l'histoire de la communauté qui la parle et de son degré de cohésion interne. Chez les peuples tribaux, « on a observé un grand émiettement territorial des langues» (Piščalnikova, Sonin 2009, p. 93), qu'on peut définir par les termes de continuum linguistique initial : chaque langue ressemble à sa voisine directe, mais moins à la suivante » (idem, p. 93). Lorsque des tribus ou des clans s'unifient, l'une des langues tribales devient dominante, se consolide : «C'est en général le dialecte de la tribu autour de laquelle naît l'unification » (Pengitov 1958, p. 243). Naturellement, les langues maries sont passées par ces différentes étapes : de dialectes de clans, elles sont devenues langues de tribus puis langues de nations. Il est possible qu'autrefois les locuteurs du mari des collines et du mari des plaines aient communiqué entre eux avec des langues moins différenciées. La formation de deux groupes distincts peut s'expliquer, en plus de raisons linguistiques, par les différentes conditions de vie des communautés et par plusieurs facteurs extralinguistiques. Aucune des deux langues maries ne s'est imposée, chaque groupe a continué et continue 
aujourd'hui de parler sa langue. Il n'y a pas eu apparition d'un standard unique qu'auraient adopté les Maris des collines et les Maris des plaines. Les sous-systèmes des deux langues maries se sont transformés, éloignant ainsi le mari des collines du mari des plaines: mutation des systèmes phonétiques, apparition ou disparition de phonèmes dans l'une ou l'autre langue; changements dans l'expression orale; transformation des types et catégories morphologiques, des structures syntaxiques. Le système des deux langues s'est fortement différencié, au point qu'il fut pratiquement impossible de former une langue littéraire marie dans les années 1920, malgré les efforts déployés pour créer une seule langue littéraire à l'orthographe unifiée (Voprosy). C'est ainsi que fut créée une seule littérature marie des collines et marie des plaines. Par la suite, la question de l'unification de l'orthographe des langues maries réapparut à plusieurs occasions. L'une des mesures concrètes pour « rapprocher » les deux langues (orthographes) dans les années 1950 fut «d'éliminer » l'harmonie vocalique du mari des collines (Asylbaev 1951). Ainsi, le mot Ӹд̈̈Р ['əдәг] « jeune fille »

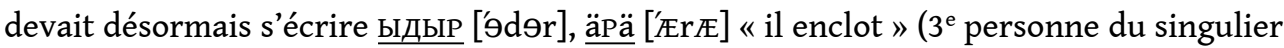
du verbe äPäШ « enclore ») devenait APA ['ArA]. Le problème est qu'en mari des collines les mots ыдыр ['Эdør] et APA ['ÁA] signifient respectivement «taupe » et «tas » et qu'ils se prononcent exactement de la même façon. La "nouvelle orthographe » eut pour unique conséquence de rendre la lecture difficile pour les Maris des collines, qui cessèrent de comprendre les textes qu'ils lisaient. L'orthographe traditionnelle fut réintroduite, le mari des collines " avait rejeté » cette ingérence extérieure artificielle. Il n'avait donc pas été possible de créer une seule langue littéraire marie à partir de deux systèmes linguistiques stables et indépendants.

8 La période «historique » du développement des deux langues littéraires maries commence avec l'apparition de textes littéraires. Si, avant l'époque soviétique, des recherches sur le mari des collines avaient été entreprises par des savants étrangers, durant l'époque soviétique et jusque dans les années 1990 il n'y eut aucune étude consacrée à cette langue. Si dans les écoles l'enseignement se faisait en mari des collines (il existait des manuels pour toutes les disciplines : mathématiques, botanique, etc.), cette langue disparaissait dans les établissements d'enseignement supérieur. Dans la littérature scientifique soviétique, on ne trouve que des observations isolées en mari des collines (Gruzov 1960, Osnovy 1976). Cela peut s'expliquer par l'absence de chercheurs connaissant le mari des collines et par le faible nombre de scientifiques issus de cette ethnie. C'est là l'origine de tous les «malheurs » du mari des collines, indépendamment du fait que son statut de langue à part entière est indiscutable.

Plusieurs générations de chercheurs ont produit des travaux scientifiques de qualité. Dans l'argumentation théorique, ils jouent un rôle premier, car la spécificité de chaque langue ne peut être comprise que par des spécialistes (Pengitov 1958).

Passons ainsi en revue quelques critères linguistiques et sociolinguistiques bien connus, utilisés pour définir ce qu'est une langue littéraire indépendante et ce qui la différencie d'un dialecte :

11 1) Une écriture normalisée. Une langue littéraire est toujours écrite (Pengitov 1958, p. 250). Un dialecte n'a pas d'écriture fixe, il n'existe que sous la forme orale. Dans la république du Mari El, les deux langues maries sont des langues littéraires, elles disposent chacune d'une écriture. Les locuteurs du mari des collines et du mari des plaines utilisent depuis une centaine d'années des formes littéraires différentes, standardisées et ayant développé une gamme de styles fonctionnels. 
12 2) Une norme réglementée. S'il y a une écriture, il va de soi que la langue littéraire obéit à un ensemble de normes précises, codifiées, à des règles phonétiques, graphiques, orthographiques et de ponctuation. La langue littéraire obéit, elle aussi, à des règles de prononciation. Ces normes accentuelles et grammaticales, l'utilisation des mots et d'autres normes concernent aussi bien la langue écrite que la langue orale.

Les deux langues maries possèdent chacune, à tous les niveaux, des caractéristiques propres (phonèmes, morphèmes, mots, groupe de mots, propositions) dues à un ensemble de facteurs internes à chacune. Le mari des collines, avec 35 phonèmes (10 voyelles et 25 consonnes), son harmonie vocalique, son accent de mot fixe, ses dix cas grammaticaux, son propre vocabulaire, est différent du mari des plaines qui ne compte que 31 phonèmes (8 voyelles et 23 consonnes), ignore l'harmonie vocalique, possède un accent de mot mobile, sept cas grammaticaux, son propre lexique, etc. En revanche, dans un dialecte, il n'y a pas de norme spécifique fixée par l'écriture. Ses traits se développent spontanément, non contraints par des règles fixes (Pengitov 1958, p. 250).

Chacune des deux langues maries possède ainsi sa norme, ses règles phonétiques, grammaticales, graphiques, orthographiques, etc.

15 3) La non-coïncidence des aires de diffusion des deux langues. Le mari des collines a toujours été parlé sur la rive droite de la Volga, plus haute, et le mari des plaines sur la rive gauche. Les échanges entre les Maris des collines et ceux des plaines ont toujours été faibles, car ces peuples n'ont pas éprouvé le besoin de communiquer de façon intensive, ni dans le passé, ni à l'époque contemporaine.

16 4) La langue comme moyen de communication. Une langue littéraire sert de moyen de communication à une communauté. Le moyen de communication des Maris des collines est le mari des collines et le russe, et le moyen de communication des Maris des plaines est le mari des plaines et le russe.

17 5) La présence d'une traduction. Un critère important pour définir le statut d'une langue par rapport à une autre est la présence ou l'absence de traductions entre ces langues. On ne traduit pas d'une langue littéraire vers un dialecte (ni d'un dialecte vers une langue littéraire) (Musorin 2001). L'existence de traductions régulières entre les langues maries témoigne de l'indépendance de ces deux langues. Il faut noter que «l'existence de traductions entre deux langues, dans les cas où l'intercompréhension entre les locuteurs de ces langues n'est pas trop difficile, est plus que tout la démonstration d'une différence " (Musorin 2001). On traduit des œuvres littéraires d'auteurs maris des plaines (J. P. Majorov-Šketan, S. G. Čavajn) vers le mari des collines et d'auteurs maris des collines (N. Ignat'ev, G. Matjukovskij, V. Suzy, etc.) vers le mari des plaines. Par ailleurs, des œuvres littéraires russes et étrangères sont traduites vers les deux langues.

18 6) L'utilisation par les locuteurs natifs d'un terme propre pour désigner leur langue (et aussi leur ethnie), différent de ceux utilisés dans les langues voisines, constitue un autre critère d'indépendance de la langue (Musorin 2001). L'existence des termes « mari des collines » (« кырык мары ») et « mari des plaines » (« олык мАРИ ») est une des manifestations de la conscience linguistique et de l'auto-identification. À partir du moment où les locuteurs d'une langue commencent à considérer leurs langues comme indépendantes et à utiliser des termes différents pour les désigner, nous pouvons affirmer que la protolangue a complètement cessé d'exister (ibidem). Plus haut a été 
citée une source dans laquelle on indiquait que déjà aux $\mathrm{XV}^{\mathrm{e}}-\mathrm{XVI}^{\mathrm{e}}$ siècles les Maris se définissaient comme étant « des collines » ou « des plaines ». La raison était sans doute due à l'existence de deux langues différentes.

Il n'existe par conséquent aucune raison de ne pas reconnaître le caractère indépendant du mari des collines. Les deux langues maries sont les représentantes légitimes de leurs locuteurs, les Maris des collines et les Maris des plaines.

De prime abord tout semble simple et compréhensible en ce qui concerne le fonctionnement des langues maries. En octobre 1995, la république du Mari El a adopté la loi sur les Langues (Zakon 1995). Sur la base de la Constitution de la Fédération de Russie du 12 décembre 1993, plusieurs républiques nationales de Russie, dont la république du Mari $\mathrm{El}$, déclarèrent leurs langues «langues d'État» au côté du russe (Konstitucija 2002). L'Article 1 de cette loi stipule : «Les langues d'État de la république du Mari El sont le mari (des collines et des plaines) et le russe... » (Zakon 1995).

21 L'adoption de la loi sur les Langues dans la république du Mari El aurait dû contribuer à créer les conditions pour le développement des deux langues et permettre les recherches scientifiques en mari des collines. Hélas, il n'en a rien été. Le contenu ambigu de l'Article $1 \mathrm{a}$ " freiné » non seulement les recherches scientifiques, mais aussi le développement du mari des collines dans son ensemble (Zakon 1995).

Un des problèmes majeurs de ce texte se trouve dans la formulation « mari (des collines et des plaines) ». Le mot «mari » ne peut pas être utilisé au singulier, car cette même loi pose qu'il s'agit de deux langues, et il ne saurait en être autrement, car la langue est l'héritage spirituel du peuple, elle en représente physiquement la substance matérielle. Il devient alors difficile de comprendre comment on peut réunir deux systèmes complètement différents (le mari des collines et le mari des plaines) en une langue abstraite (la « langue marie ») qui n'existe pas.

Le terme " (langue) marie » (au singulier) a semé la confusion dans l'opinion publique. Les gens ont du mal à comprendre ce que cela signifie, et c'est la raison pour laquelle sont nées des interprétations différentes. Les uns se représentent la "langue marie » comme une «macro-langue » englobant le mari des plaines et le mari des collines. Ils pensent par conséquent que tous les Maris des collines parlent en mari des plaines (Gabelents 1841). La réalité est que les Maris des collines n'ont jamais parlé et ne parlent pas le mari des plaines, tout comme les Maris des plaines n'ont jamais parlé et ne parlent pas le mari des collines. D'autres pensent qu'une "langue marie » est réellement utilisée sur le territoire de la république du Mari El, ils propagent le concept de « langue marie ». Pour certains d'entre eux, il s'agit uniquement du mari des plaines, ce dont témoignent les travaux de nombreux chercheurs. Certains scientifiques ne remarquent même pas les rapports systémiques dans les langues maries et décrivent tels ou tels phénomènes linguistiques "en mettant tout dans le même sac ». Il y en a aussi qui qualifient de façon tout à fait arbitraire le mari des collines de dialecte (mais on ne sait pas de quelle langue). Une troisième catégorie de personnes appelle à tort le mari des plaines « langue marie proprement dite » alors que ces termes conviendraient davantage au mari des collines, dans la mesure où celui-ci a mieux conservé que d'autres langues finno-ougriennes plusieurs traits de la protolangue: riche système vocalique, harmonie vocalique, présence rare de certaines combinaisons phonétiques, riche système casuel ${ }^{2}$, etc. emploie-t-elle cette formulation malgré l'existence évidente et réelle de deux langues 
indépendantes ? La "réponse » peut être entendue ou lue relativement souvent : « ... afin d'élever le rôle de la langue marie dans l'affirmation ethnique du peuple ». C'est justement cette opinion subjective qui est la base de l'adoption de la loi sur les Langues: chez «le» peuple mari ne doit exister qu'une seule langue; l'autoidentification de chacun doit être la même : tous doivent s'appeler «Maris ", car tous les Maris se comprennent.

Ces positions témoignent de la maîtrise insuffisante, voire inexistante, des connaissances scientifiques nécessaires à la définition d'une langue, de l'autoidentification ou de la conscience ethnique. Il faut au contraire s'appuyer sur les faits, sur la logique, sans idées préconçues ni déductions dictées par la conjoncture politique.

Examinons ce que signifie le critère « un peuple = une langue ». Il est inapproprié, car la définition d'un peuple est encore plus compliquée que la définition d'une langue. De plus, on observe régulièrement l'absence de correspondance entre peuples et langues : par exemple, le peuple dit « soviétique » parlait le russe, mais aussi plus de 130 langues différentes ; les Mordves parlent le mokcha, l'erza et le russe ; les Maris parlent le mari des collines, le mari des plaines et le russe. Dans le monde, on trouve des exemples de peuples plurilingues, ou des peuples différents parlant la même langue. L'identité entre langue et peuple est plutôt rare. Il est évident que l'un des paramètres pour l'identification des peuples est le paramètre linguistique, et non le contraire.

Sur «l'auto-identification des locuteurs natifs", on peut noter les points suivants : de nombreux enquêteurs se reposent entièrement sur l'opinion des locuteurs (probablement est-ce le cas des organisateurs des recensements de 2002 et 2010 dans la république du Mari El). Cependant, l'opinion de locuteurs différents peut ne pas coïncider ; dans de nombreuses cultures, les personnes n'ont jamais eu et n'ont pas du tout de représentation nette de leur langue. C'est pourtant précisément la langue qui exprime la conscience de l'individu. Avoir une conscience linguistique, c'est se représenter la langue que l'on parle. Si un groupe de locuteurs considère que sa langue maternelle est différente de celle de ses voisins, cela signifie que la langue parlée par ce groupe est différente et indépendante (Musorin 2001). Il ne fait aucun doute que les Maris des plaines considèrent leur langue comme étant différente de celle des Maris des collines, et que les Maris des collines considèrent leur langue comme différente de celle des Maris des plaines.

Les recherches de l'Institut d'Ethnologie et d'Anthropologie RAN, menées sous la direction du professeur V.V.Pimenov, en témoignent: "Les Maris des collines s'identifient assez nettement dans leur conscience nationale avec l'ethnonyme « КЫРЫК МАРЫ». Les Maris des plaines - «олЫК МАРИ", nettement supérieurs en nombre, sont dispersés sur tout le territoire situé sur la rive gauche de la Volga, ce qui explique les particularités locales de leur culture et de leur langue, qui créent chez eux une identité plurielle. Les Maris des plaines s'identifient souvent simplement comme des « Maris », tout en ayant bien conscience de leur appartenance locale $»^{3}$.

Sur "l'intercompréhension des langues» ou le fait que les Maris des collines et les Maris des plaines se comprennent dans la vie de tous les jours, il ne faudrait pas oublier que si l'absence d'intercompréhension peut témoigner qu'on a affaire à des langues différentes, l'intercompréhension ne signifie pas forcément que nous sommes en présence de dialectes. Les locuteurs de certaines langues slaves (russe, biélorusse, ukrainien) ou de certaines langues turciques (tatar, kirghiz) sont tout à fait capables dans la vie quotidienne de se comprendre sans traducteur, mais personne ne songerait 
à considérer le biélorusse, l'ukrainien et le russe, ou le tatar et le kirghiz comme les dialectes d'une seule langue slave ou turcique (Popova-Sterin 2007).

Le critère de l'« intercompréhension" s'applique le plus souvent aux langues sans écriture. En guise d'exemple d'intercompréhension entre Maris des collines et Maris des plaines, observons quelques résultats des recherches effectuées au Laboratoire de Linguistique expérimentale et appliquée de l'Université d'État du Mari El. Les expériences témoignent d'un niveau plutôt bas de l'indice d'intercompréhension entre ces deux groupes. Le matériel utilisé pour ces recherches était le Dictionnaire fréquentatif $d u$ mari des collines et $d u$ mari des plaines ainsi que des textes publicitaires (Statističeskij 1998). Les Maris des collines devaient identifier le lexique connu et inconnu dans une liste de mots en mari des plaines, et les Maris des plaines devaient faire le même exercice avec une liste de mots en mari des collines. Dans la liste des mots en mari des collines commençant par « $\mathrm{S}$ ", les Maris des plaines en ont reconnu $36 \%$. Dans la liste des mots en mari des plaines commençant par « $S$ », les Maris des collines en ont reconnu $42 \%$. De façon générale, pour les locuteurs des deux langues, l'«intercompréhension» est de l'ordre de 38 à $40 \%$. Cette intercompréhension est grandement « facilitée » par le nombre de mots empruntés au russe.

31 Selon l'Institut d'ethnologie et d'anthropologie de l'Académie des sciences de Russie, «l'existence de deux normes littéraires indépendantes est due aux importantes différences lexicales et, dans une moindre mesure, grammaticales. Les différences phonétiques entravent elles aussi l'intercompréhension linguistique entre Maris des collines et Maris des plaines. Pour cette raison, comme le montrent les recherches ethnosociolinguistiques, ces deux communautés préfèrent six fois sur dix utiliser le russe pour communiquer entre elles » (Častotnyj 2005).

D'une façon générale, les critères sociolinguistiques ne peuvent pas toujours être les causes des différences linguistiques et de l'identification des locuteurs. Comme on l'a $\mathrm{vu}$, dans le cas des langues maries, ce sont surtout les critères linguistiques qui permettent le plus clairement et avec le plus d'objectivité de savoir s'il s'agit d'une ou de plusieurs langues. Dans la république du Mari El, la situation linguistique réelle est qu'il y a aujourd'hui deux langues littéraires maries indépendantes et équivalentes. L'ambiguïté de la formulation de la loi sur les Langues dans la république du Mari El se reflète dans la vie sociopolitique de la République, mais pas dans la loi. Selon le droit social, la loi « obligerait-elle » les Maris des collines et les Maris des plaines à parler une langue marie abstraite qui n'existe pas?

Ainsi, l'idée selon laquelle "un peuple = une langue", à la base de la loi, s'est manifestée de façon grotesque lors des recensements de 2002 et de 2010, quand on a interdit aux Maris des collines de mentionner la nationalité "Mari des collines" (Anikina 2011, Respublika 1999, Respublika 2003). À la question « Pourquoi ne peut-on pas écrire cela? ?, les copistes répondaient : «Ce sont les directives reçues ».

Lors de la préparation du recensement, les copistes se sont demandé dans quels cas ils devaient indiquer "Maris des collines", "Maris des plaines» ou tout simplement "Maris ». On leur a expliqué qu'il était strictement interdit, lors du recensement, de donner des conseils, de faire la moindre allusion ou d'imposer leurs propres opinions. Ainsi, lors du recensement, les citoyens avaient le droit de s'exprimer librement, donnant parfois des réponses tout à fait ridicules, qui étaient ensuite corrigées de façon appropriée lors du traitement des données. Pour la première fois en Russie, ces recensements auraient dû se dérouler conformément aux normes internationales et 
aux recommandations de l'ONU. Les copistes en république du Mari El se sont visiblement trouvés face à des situations difficiles, jamais rencontrées auparavant dans les statistiques nationales.

Selon le recensement de 2002, il y avait en tout et pour tout 17715 Maris des collines [Anikina 2011, Respublika 1999). En 1989, les Maris des collines s'élevaient à 44296 personnes (Popova, Sternin 2007, Respublika 2004). D'un recensement à l'autre, 26581 personnes ont tout simplement "disparu» sans laisser de traces (nous ne parlons pas ici de la baisse démographique générale, qui ne se manifeste pas du tout avec des chiffres aussi énormes). Tout le monde sait que dans le raïon des Maris des collines vivent des Maris des collines qui parlent le russe et le mari des collines; les Maris qui vivent dans les raïons de Kilemary et de Jurinskij parlent aussi le mari des collines. Le service territorial des statistiques d'État en république du Mari El justifie le chiffre de 17715 de la façon suivante: les Maris des collines sont tout simplement devenus des "Maris», par un processus «d'auto-identification». Si les Maris des collines vivant en périphérie de leur zone d'habitation peuvent en effet se qualifier simplement de « Maris », ceux qui vivent dans la République se définissent clairement comme « Maris des collines » et ne s'identifient en aucune façon aux Maris des plaines. De même, les Maris des plaines ne s'identifient pas aux Maris des collines.

36 Selon les données du recensement de 2010, les Maris des collines sont au nombre de $23502^{4}$. Doit-on en conclure qu'entre 2002 et 2010, les Maris des collines ont augmenté de 5787 ? Ces dernières années, dans toute la République, on observe une tendance à l'augmentation du taux de naissances. Quoi qu'il en soit, 17715 personnes n'ont pu donner en huit ans naissance à 5787 enfants !

Il est probable que les résultats auraient été différents si les organisateurs du recensement avaient tenu compte $d u$ critère linguistique pour définir l'autoidentification, et s'ils avaient suivi un procédé plus authentique et adéquat pour étudier la dynamique des langues et de la société dans son ensemble.

Comme on le voit, c'est justement le caractère ambigu de la formulation de la loi sur les Langues qui a entraîné, dans les cas des recensements de 2002 et de 2010, une politique agressive et assimilatrice stipulant qu'en république du Mari El ne doivent vivre que des "Maris", en conformité avec l'adage (plus du tout actuel) "un peuple = une langue ». Cette politique s'efforce aussi de montrer la puissance démographique d'un groupe linguistique et la faiblesse de l'autre, avec l'objectif que la langue d'État (ici il s'agit de la langue ethnique) soit la langue de la majorité (par rapport au nombre d'habitants). De telles positions auraient pu être permises dans la période "préhistorique », quand les langues maries n'avaient pas encore de littérature. Aujourd'hui, alors que deux langues littéraires existent, l'idée de créer une seule langue marie est tout simplement inacceptable, car synthétiser artificiellement deux systèmes linguistiques est pratiquement impossible, tout comme il est impossible d'interdire pour des raisons politiques l'utilisation de l'une ou de l'autre langue à une étape donnée de leur développement social. Il est étrange d'affirmer que l'existence de deux cultures et de deux langues ne serait pas une bonne chose: dans notre République il n'y a aucune animosité entre les peuples. La présence de deux langues maries devrait être considérée comme une richesse culturelle et historique, car ces deux langues, avec leur écriture et leur littérature, font partie de l'histoire et de la civilisation.

39 Autre problème de l'Article 1 de la loi : le mot «État 》 (Les langues d'État de la république du Mari El sont le mari (des collines et des plaines) et le russe) (Zakon 1995). 

écrit : « 1 . Le russe est la langue officielle de la Fédération de Russie sur l'ensemble de son territoire. Les Républiques ont le droit d'instituer leurs propres langues officielles. Celles-ci peuvent être utilisées, à côté du russe, dans les organes du pouvoir de l'État, dans les collectivités locales, dans les administrations d'État des Républiques... 3. La Fédération de Russie garantit à tous ses peuples le droit de préserver leurs langues maternelles et la création des conditions pour leur enseignement et leur développement »(Konstitucija 2002).

41 Selon la Constitution de la Fédération de Russie du 12 décembre 1993, chaque entité nationale peut conduire les affaires de l'État dans sa propre langue, les langues autochtones peuvent devenir des langues d'État. Les politiques nationales n'ont cependant pas pris en compte le fait que le russe, de par son extension, écrase les langues nationales (dans notre cas les langues maries), et que toutes les langues autochtones ne possèdent pas un niveau de développement leur permettant de devenir des langues d'État.

loi sur les Langues dans la république du Mari El les langues maries sont appelées langues d'état, ce qui implique qu'elles devraient remplir toutes les fonctions correspondant à des langues d'État.

dén poids réel dans la société où elle est parlée définissent la sphère de diffusion de cette langue et le volume de ses fonctions (Piščalnikova, Sonin 2009, p. 360): le fonctionnement d'une langue quelconque dépend de son niveau de développement et de son utilisation dans la société.

fonctions d'une langue d'État se manifestent à travers différents types de textes : textes officiels, techniques, scientifiques, publicitaires, artistiques, informatifs... D'ailleurs, l'existence de différents types de textes est un bon indicateur du niveau de développement d'une langue.

es textes officiels concernent les questions diplomatiques, législatives et administratives. Le russe, en tant que langue officielle, est utilisé dans tous ces textes. Il n'y a pas de textes diplomatiques dans les langues maries, et les textes législatifs ou administratifs ne sont que des traductions du russe: il s'agit le plus souvent d'attestations de naissance, de décès et de mariages en mari des plaines. Il faut signaler ici que la traduction de tels documents du russe vers le mari des collines n'est guère envisagée. On donne aux Maris des collines des attestations traduites du russe en mari des plaines. Les traductions de ces textes ne servent pas à la fonction communicative des langues maries.

scientifiques écrits dans les langues maries sont extrêmement limités et se limitent à la linguistique. Il existe des manuels de langues pour les écoles et pour les spécialistes (qui travaillent sur le mari des plaines) de l'Université d'État marie. Dans les écoles secondaires et dans les établissements d'enseignement supérieur de la République, l'enseignement se fait en russe; l'une ou l'autre langue marie n'y est qu'une matière parmi d'autres.

47 On peut donc constater qu'il n'y a pratiquement pas de matériau informatif, ni de textes techniques, en langues maries.

48 Les langues maries sont plus ou moins largement utilisées dans le domaine culturel, grâce aux textes publicitaires et d'information. Il faut toutefois noter que dans les

Études finno-ougriennes, 45 | 2013 
conditions économiques actuelles, l'édition de littérature en langues maries s'est considérablement réduite.

De cette façon, les langues maries sont fonctionnellement limitées à la vie sociale. Les deux langues sont à la base des échanges quotidiens et elles s'utilisent en partie dans la sphère artistico-publicitaire.

La présence limitée des langues maries dans la vie politique et publique de la république du Mari El s'explique d'un côté par la limitation de leur développement fonctionnel, de l'autre par le manque de nécessité de leur emploi dans l'administration. Le statut des langues maries comme langues d'État n'est que déclaratif, dans la réalité ce sont des langues autochtones régionales. Comme on le voit, les démocrates russes se sont empressés de leur donner le statut de «langues d'État ». Cette idée, excellente idée en soi, était hélas impossible à réaliser.

51 La définition précise du statut des langues autochtones comme langues régionales et nationales aurait pu être un moyen utile pour résoudre la question de leur préservation dans les conditions actuelles de la mondialisation.

52 La «fragilité » de la culture et de la vie des langues des petits peuples, en particulier des Maris des collines et des Maris des plaines, est très perceptible. Des années 1920 aux années 1960 environ, dans les écoles maries, l'enseignement de toutes les matières se faisait en langues maries (en mari des collines ou en mari des plaines selon les aires de peuplement des Maris). Il y avait assez de financement pour permettre l'édition de journaux, de magazines, de publications d'écrivains et de poètes.

53 À partir des années 1960 commence une période de revirement dans l'histoire du développement des langues nationales: le russe devient la langue du peuple et la langue de l'enseignement dans les écoles; les langues maries n'y sont enseignées que comme matière (cela dure déjà depuis 50 ans). C'est la raison pour laquelle il y a de moins en moins de lecteurs pour les livres publiés en langues vernaculaires, ce dont témoigne l'engorgement des livres invendus dans les maisons d'édition. À partir de cette période et sous l'influence du russe - de plus en plus présent - d'importants changements interviennent dans les systèmes des deux langues maries. Selon le Laboratoire de linguistique expérimentale et appliquée de l'Université d'État du Mari El, dans le lexique du mari des plaines contemporain on compte environ $34 \%$ d'emprunts russes, dans le lexique du mari des collines environ $55 \%$. Dans l'ensemble, l'analyse de la situation contemporaine des langues maries fait ressortir une transformation réelle et intensive du lexique et de la phonologie, mais aussi du système sémantico-stylistique, sous l'influence du russe. Du point de vue de leur développement - résultat d'une influence extérieure - on peut affirmer que les deux langues se trouvent au stade où de moins en moins de locuteurs éprouvent le besoin de les utiliser dans la société.

Pour une définition plus précise du danger qui menace les langues, le Livre rouge de l'UNESCO a inventorié une échelle de six catégories. Sur cette échelle, le mari des collines est situé parmi les langues "sérieusement menacées" (pour qu'une langue se conserve, il faut environ 100000 locuteurs) et le mari des plaines se trouve parmi les langues «menacées ». Il est intéressant de noter que les Nenetses (44 640 personnes selon le recensement de 2010), les Evenks (37 843), les Khantys (30 943) et d'autres peuples encore sont classés parmi les peuples autochtones de Russie à faible population. Mais les Maris des collines (23 502) ne sont-ils pas aussi un peuple 
autochtone? La prise de conscience de cette situation exige la mise en place de programmes adéquats pour la documentation et la préservation des langues maries.

D'une manière générale, nous pensons que l'une des clés de l'élaboration de la politique linguistique dans la république du Mari El et dans toute la Russie reste la question de la définition du statut des langues nationales, dont la solution exige la rigueur, la précision et l'honnêteté de tous les responsables de sa mise en œuvre.

\section{BIBLIOGRAPHIE}

AL’BINSKIJ 1837 = АЛЬБИНСКИЙ А., ЧЕРЕМИССКАЯ ГРАММАТИКА, КАЗАНЬ: УНИВ. ТИП.

ANIKINA 2011 = АНИКИНА А., «ЕЗИДЫ, УЛЬЧИ, КАРАКАЛПАКИ И УЙГУРЫ УЖИВАЮТСЯ В МАРИЙ ЭЛ», ГАЗЕТА «РРоГОРОД», №11 (79), 19/3, с. 16-17.

ASYLBAEV 1951 = АСЫЛБАЕВ А.А., «ВОПРОСЫ РАЗВИТИЯ МАРИЙСКОГО ЛИТЕРАТУРНОГО ЯЗЫКА», НАУЧНАЯ СЕССИЯ ПО ВОПРОСАМ РАЗВИТИЯ МАРИЙСКОГО ЛИТЕРАТУРНОГО ЯЗЫКА: ТЕЗ. ДОКЛ., ЙОШКАРолА, с. 3-9.

CASTRÉN Mattias Aleksanteri, 1845, Elementa grammatices tscheremissae, Kuopio.

С̌АSTOTNYJ 2005 = ЧАСТОТНЫЙ СЛОВАРЬ ГОРНОМАРИЙСКОГО И ЛУГОМАРИЙСКОГО ЯЗЫКОВ, ЙОШКАР-ОЛА: МАРГУ.

DUHOVNAJA 1769 = ДУХОВНАЯ ЦЕРЕМОНИЯ, ПРОВОДИВШАЯСЯ ВО ВРЕМЯ... ПРИСУДСТВИЯ ЕКАТЕРИНЫ ВТОРЫЯ В КАЗАНЕ, С ПРИЛОЖЕНИЕМ ПРИ ТОМ СЛОВОМ О НЕСРАВНЕННОМ ВЕЛИКОДУШИИ... ИМП. САМОДЕРЖИЦЫ ВСЕРОССИЙСКАЯ, ПОЛУЧИВШАЯ БЛАГОПОЛУЧНОЕ ОТ ПРИВИВАНИЯ ОСПЫ ВЫЗДОРОВЛЕНИЕ, СПБ.: ПРИ ИМП. АКАД. НАУК.

EVANGELIE 1821 = ЕВАНГЕЛИЕ НА ГОРНОМ НАРЕЧИИ, САНКТ-ПЕТЕРБУРГ.

GABELENTS Hans Conon von, 1841, „Vergleichung der beiden tscheremissischen Dialekte“, Zeitschrift für die Kunde des Morgenlandes, Heft 1, S. 122-139.

GRUZOV 1960 = ГРУЗОВ Л.П., СОВРЕМЕННЫЙ МАРИЙСКИЙ ЯЗЫК: ФОНЕТИКА, ЙОШКАР-ОЛА: МАРКНИГОИЗДАТ.

KONSTITUCIJA 2002 = КОНСТИТУЦИЯ РОССИЙСКОЙ ФЕДЕРАЦИИ, МОСКВА: ИЗДАТЕЛЬ УМЕРЕНКОВ.

LEWIS M. Paul (ed.), 2009, Ethnologue: Languages of the World, 16th edition, ethnologue.com.

MUSORIN 2001 = мУСОРИН А. Ю., «ЧТО ТАКОЕ ОТДЕЛЬНЫЙ ЯЗЫК?» (СИБИРСКИЙ ЛИНГВИСТИЧЕСКИЙ

СЕМИНАР. № 1), НОВОСИБИРСК, 2001, № 1, С. 12-16.

OSNOVY 1976 = ОСНОВЫ ФИННО-УГОРСКОГО ЯЗЫКОЗНАНИЯ: МАРИЙСК., ПЕРМ. УГОР. ЯЗЫКИ, МОСКВА: НАУКА.

PENGITOV 1958 = ПЕНГИТОВ Н.Т., СОПОСТАВИТЕЛЬНАЯ ГРАММАТИКА РУССКОГО И МАРИЙСКОГО ЯЗЫКОВ, Ч. 1: ВВЕДЕНИЕ, ФОНЕТИКА, МОРФОЛОГИЯ, ЙОШКАР-ОЛА: МАРКНИГОИЗДАТ. 
PIŠČAL'NIKOVA, SONIN 2009 = ПИщАЛЬНИКОBA В.А., СОНИН А.Г., ОБЩЕЕ ЯЗЫКОЗНАНИЕ: УЧЕБНИК ДЛЯ СТУД. ВЫСШ. УЧЕБ. ЗАВЕДЕНИЙ, МОСКВА: ИЗДАТЕЛЬСКИЙ ЦЕНТР «АКАДЕМИЯ». POPOVA, STERNIN 2007 = ПОПОВА З.Д., СТЕРНИН И.А,. ОБЩЕЕ ЯЗЫКОЗНАНИЕ. УЧЕБНОЕ ПОСОБИЕ, 2-Е ИЗД., ПЕРЕРАБ. И ДОП., МОСКВА: АСТ ВОСТОК-ЗАПАД.

RAMSTEDT Gustaf John, 1902, Bergtscheremissische Sprachstudien, Helsingfors.

RESPUBLIKA 1999 = РЕСПУБЛИКА МАРИЙ ЭЛ. СТАТИСТИЧЕСКИЙ СБОРНИК. Ч.1, ЙОШКАР-ОЛА.

RESPUBLIKA 2003 = РЕСПУБЛИКА МАРИЙ ЭЛ В ЦИФРАХ. СТАТИСТИЧЕСКИЙ СБОРНИК, ЙОШКАР-ОЛА.

RESPUBLIKA 2004 = РЕСПУБЛИКА МАРИЙ ЭЛ. СТАТИСТИЧЕСКИЙ ЕЖЕГОДНИК. ЧАСТЬ 1, ЙОШКАР-ОЛА: ТЕРРИТОРИАЛЬНЫЙ ОРГАН ФЕДЕРАЛЬНОЙ СЛУЖБЫ ГОСУДАРСТВЕННОЙ СТАТИСТИКИ ПО РЕСПУБЛИКЕ МАРИЙ ЭЛ.

STATISTIČESKIJ 1998 = СТАТИСТИЧЕСКИЙ ЕЖЕГОДНИК РЕСПУБЛИКИ МАРИЙ ЭЛ. Ч.1, ЙОШКАР-ОЛА: ГОСКОМСТАТ РЕСП. МАРИЙ ЭЛ, ГОСКОМСТАТ РОССИИ, С. 129

VOPROSY = ВОПРОСЫ УЛУЧШЕНИЯ МАРИЙСКОГО АЛФАВИТА И ОРФОГРАФИИ. ЛИСТОК 2 (АРХИВНЫЕ МАТЕРИАЛЫ МАРНИИЯИЛ ИМ. В.М. ВАСИЛЬЕВА).

WIEDEMANN Ferdinand Johann, 1847, Versuch einer Grammatik der tscheremissischen Sprache: Nach dem in der Ewangelien ubersetzung von 1821 gebrauchten dialekte, Reval.

ZAKON 1995 = «ЗАКОН РЕСПУБЛИКИ МАРИЙ ЭЛ «О ЯЗЫКАХ В РЕСПУБЛИКЕ МАРИЙ ЭЛ» ОТ 26 ОКТЯБРЯ 1995 Г.», СОБРАНИЕ ЗАКОНОДАТЕЛЬСТВА РЕСПУБЛИКИ МАРИЙ ЭЛ, 8 (361).

\section{NOTES}

1. http://www.gosarh.arh-mari.ru

2. коГДА умИРАЮт ЯзыкИ. http://www.bigbook.ru/litnews/detail.php?ID=3315 (consulté le 15.03.2011).

3. КОГДА УМИРАЮТ ЯзЫкИ. http://www.bigbook.ru/litnews/detail.php?ID=3315 (consulté le 15.03.2011).

4. http://www.gosarh.arh-mari.ru

\section{RÉSUMÉS}

Cet article se concentre sur les Maris des collines et leur langue. Il en retrace l'histoire et évoque le processus qui a abouti à la constitution de deux langues littéraires maries, argumentant sur les raisons de considérer le mari des collines comme une langue à part entière et soulignant les problèmes que pose la formulation ambiguë du passage de la loi sur les Langues de la République marie. Finalement, il s'interroge sur l'usage actuel du mari des collines dans la vie de la communauté. 


\section{INDEX}

Index géographique : Fédération de Russie, Républiques finno-ougriennes, Mari-El (République), Koz'modem'jansk, Pertnury, Kilemar, Jurinskij, Républiques turcophones, Tatarstan (République), Tchouvachie (République), Kazan, Čeboksary, Saint-Pétersbourg

Keywords : Auto-identification, language awareness, language planning, language status, language codification, Hill Mari, Meadow Mari, Russian, Bielorussian, Ukrainian, Tatar, Kirghiz Mots-clés : auto-identification, conscience linguistique, aménagement linguistique, statut linguistique, codification linguistique nomsmotscles Maris des collines, Maris des plaines, Russes, Tatars motsclesru САМОИДЕНТИФИКАЦИЯ, ЯЗЫКОВОЕ СОЗНАНИЕ, РЕГЛАМЕНТИРОВАННАЯ НОРМИРОВАННОСТЬ, СТАТУС ЯЗЫКА

Thèmes : linguistique

disciplines biélorusse, russe ukrainien, tatar, kirghiz 